\title{
Can BAP1 expression loss in mesothelial cells be an indicator of malignancy?
}

\author{
Hanife Gulnihal Ozdemir ${ }^{1}$, Sermin Coban Kokten², Nagehan Ozdemir Barisik ${ }^{2}$ \\ 'Department of Pathology, Osmaniye Public Hospital, Osmaniye; 2University of Health Sciences Kartal Dr Lutfi Kirdar Training and Research Hospital, Istanbul, Turkey
}

\begin{abstract}
Background: Malignant mesothelioma is a highly aggressive tumor that can be confused with a benign mesothelial lesion, especially cytomorphologic lesions. BRCA1-associated protein 1 (BAP1) acts as a tumor suppressor. In this study, we aim to investigate the value of BAP1 staining of malignant mesothelioma cases with expression loss and diagnosis in cell block and biopsy tissue. Methods: Between January 2009 and March 2017, 64 mesotheliomas, 117 reactive mesothelial hyperplasias, and 20 fibrinous pleuritis/pericarditis were diagnosed with morphologic and immunohistochemical findings in our pathology clinic and were included in the study. Formalin-fixed, paraffin-embedded tissues were immunohistochemically examined for BAP1. Inflammatory and stromal cells were used as positive internal controls. BAP1 was assessed for nuclear staining in mesothelial cells. Results: Examinations of the relationship between patient diagnosis and BAP1 biopsy status showed that the BAP1 loss rate $(76.6 \%)$ was significantly higher in malignant mesothelioma cases than in other benign diseases $(0 \%)(p<.001)$. Sensitivity and specificity were $76.56 \%$ and $100 \%$, respectively, for biopsy tissue from malignant mesothelioma. Sensitivity and specificity were both $100 \%$ for BAP1 test on cell block tissue. Furthermore, the consistency between BAP1 cell block and biopsy results was excellent $(\mathrm{k}=0.90)$ and the correlation was significant $(\mathrm{p}<.001)$. Conclusions: This study shows that BAP1 expression loss in both cytology and biopsy tissue in biopsy-confirmed malignant mesothelioma cases is an essential parameter for malignant mesothelioma diagnosis.
\end{abstract}

Key Words: Asbestosis; BAP1; Immunohistochemistry; Malignant mesothelioma; Pleura

Received: May 26, 2020 Revised: August 22, 2020 Accepted: September 14, 2020

Corresponding Author: Hanife Gulnihal Ozdemir, MD, Department of Pathology, Osmaniye Public Hospital, Akyar Central Location Hospital Street Osmaniye/Center 80010, Turkey Tel: +90-5547884275, E-mail: dr.gulnihalzdmr@gmail.com

Malignant mesothelioma (MM) is an aggressive tumor originating from serosal surfaces, mostly pleura [1]. Exposure to some carcinogens, especially asbestos, is a strong risk for MM development [2,3]. MM has an inadequate response to treatment because in most patients diagnosis is delayed. However, prognosis is better if recognized earlier. Therefore, it is crucial to diagnose MM early [4-6].

About $80 \%$ of MM cases are associated with asbestos exposure. MM incidence is high in Turkey, especially the Cappadocia region, where environmental asbestos is widely distributed [7]. It is thought that 20 years or more of asbestos exposure can increase the risk of MM development.

Malignant mesothelioma, benign mesothelial neoplasia, and reactive mesothelial proliferation are lesions that are difficult to diagnose because they mimic each other cytomorphologically. Although the features supporting malignancy include clear cy- tological atypia, dense cell clusters, and necrosis, the most reliable diagnostic criterion is the presence of deep-tissue invasion [8]. MM diagnosis in cytologic materials is even more difficult because invasion is the only reliable standard.

To date, an immunohistochemical marker that can reliably differentiate a reactive process from neoplastic mesothelioma does not exist $[9,10]$. Various immunohistochemical markers (epithelial membrane antigen, p53, p100, p-glycoprotein, desmin, etc.) are used together to reach a final decision [11-13].

BRCA1-associated protein 1 (BAP1) acts as a tumor suppressor and belongs to the family of high-risk cancer-related genes located at 3p21.1. It is associated with a high-risk cancer syndrome that includes malignancies such as malignant mesothelioma, uveal melanoma, cutaneous melanoma, atypical melanocytic tumor, and renal cell carcinoma (especially clear-cell type). BAP1 expression loss may be an indicator of malignancy in mesotheli- 
omas $[4,14,15]$. BAP1 is not yet routinely used as an immunohistochemical marker but may soon be preferred for diagnosis and targeted treatment $[16,17]$.

This study aims to investigate the efficacy of immunohistochemically stained BAP1 antibodies for differential diagnosis of $\mathrm{MM}$, reactive mesothelial hyperplasia (RMH), and fibrinous pleuritis/pericarditis (FP).

\section{MATERIALS AND METHODS}

\section{Case selection}

Sixty-four patients with mesothelioma, 117 patients with reactive mesothelial hyperplasia, and 20 patients with FP who were diagnosed at our pathology clinic between January 2009 and March 2017 were included in the study. Diagnosis of the MM cases was made according to presence of deep-tissue invasion composed of mesothelial cells using immunohistochemically applied calretinin, WT1, and D2.40 stains of biopsy tissue. The cell blocks obtained from cytological material taken during biopsy were evaluated for the presence of mesothelial cells. The cell blocks, which were also stained with the same immunohistochemical markers, were also analyzed for this study.

We examined slides, paraffin blocks, and report archives of all the study cases where available. Cell blocks of cytological material and biopsy tissue were evaluated for suitability for immunohistochemical examination. Both cell blocks and tissue blocks that had sufficient mesothelial cells ( $\geq 20$ cells) were immunohistochemically stained with BAP1 antibodies. For cases that did not have cell blocks or that had insufficient mesothelial cells in cell blocks, only tissue blocks were stained. Two pathologists, blinded from the diagnosis, evaluated results on light microscopy.

\section{Immunohistochemical procedure}

For immunohistochemical examination, 4- $\mu$ m-thick sections prepared from formalin-fixed and paraffin-embedded tissues were used. Tissue sections were taken into electrostatically charged slides (Isotherm) and dried at $70^{\circ} \mathrm{C}$ for at least 1 hour. The whole immunohistochemical staining process, including deparaffinization and antigen release, was performed on a fully automated immunohistochemistry staining device (Ventana BenchMark XT, Ventana Medical Systems, Tucson, AZ, USA). We used a ready-made kit that is biotin-free, based on an horseradish peroxidase (HRP) multimer, and contains hydrogen peroxide substrate and 3,3'-diaminobenzidine tetrahydrochloride (DAB) chromogen (Catalog number 760-500, ultraView Universal DAB Detection Kit, Ventana Medical Systems) for this process. BAP1 antibody (1:100, Thermo Fisher Scientific, Rockford, IL, USA) was administered. Contrast staining with hemotoxylin and bluing solution was performed with a staining device; the procedure was completed manually with dehydration, transparency with xylene, and closure of the sections with lamel.

Inflammatory and stromal cells in the cell block of cytologic materials and biopsy materials were used as a positive internal control for BAP1 antibody. Mesothelial cells were evaluated via nuclear staining with BAP1 antibodies. Detection of nuclear staining was interpreted as "BAP1 normal expression" (Fig. 1AD) and lack of staining as "loss of BAP1 expression" (Fig. 2A-D). Cytoplasmic staining, observed in some cases, was evaluated as nonspecific, and we took into account only nuclear staining.

\section{Statistical analyses}

Statistical analyses were performed using SPSS ver. 16.0 software (SPSS Inc., Chicago, IL, USA). Variables were evaluated for normality both visually (histogram and probability graphs) and statistically (Kolmogorov-Smirnov/Shapiro-Wilk tests). Descriptive analyses were performed using mean and standard deviations for normally distributed variables. In cases where the data were not normally distributed, the Mann-Whitney U test was used to compare two groups and the Kruskal-Wallis test was used to compare more than two groups. The chi-square and Fisher exact tests were used to compare nominal variables, e.g., biopsy results. Patient age was recategorized into ordinal groups and trend analysis was performed with meaningful parameters. Also, we used the Kappa test to determine the consistency of biopsy results with each other. A p-value $<.05$ was considered statistically significant.

\section{RESULTS}

Of $64 \mathrm{MM}$ cases, 40 (62.5\%) were male and 24 (37.5\%) were female. The median age at diagnosis was $61( \pm 12.26)$ years, and $56.3 \%$ of the patients were between $40-64$ years old. While 57 of the 64 cases $(89.1 \%)$ originated in the pleura, six $(9.4 \%)$ were from the peritoneum and one (1.5\%) from the pericardium. In histological subtypes, 54 cases (84.4\%) were epithelioid type, five cases $(5.2 \%)$ sarcomatoid type, and 5 cases (5.2\%) biphasic type. Asbestos exposure was detected in 35.9\% of our MM cases.

Seventy-four of $117 \mathrm{RMH}$ cases (63.2\%) and 15 of $20 \mathrm{FP}$ cases (75\%) were male, with median ages of $45( \pm 19.36)$ years and $59.5( \pm 16.07)$ years, respectively. Of the 137 non-MM cases (117 RMH and $20 \mathrm{FP}), 103$ biopsy specimens (75.1\%) were taken from the pleura, 31 (22.6\%) from the peritoneum, and 3 

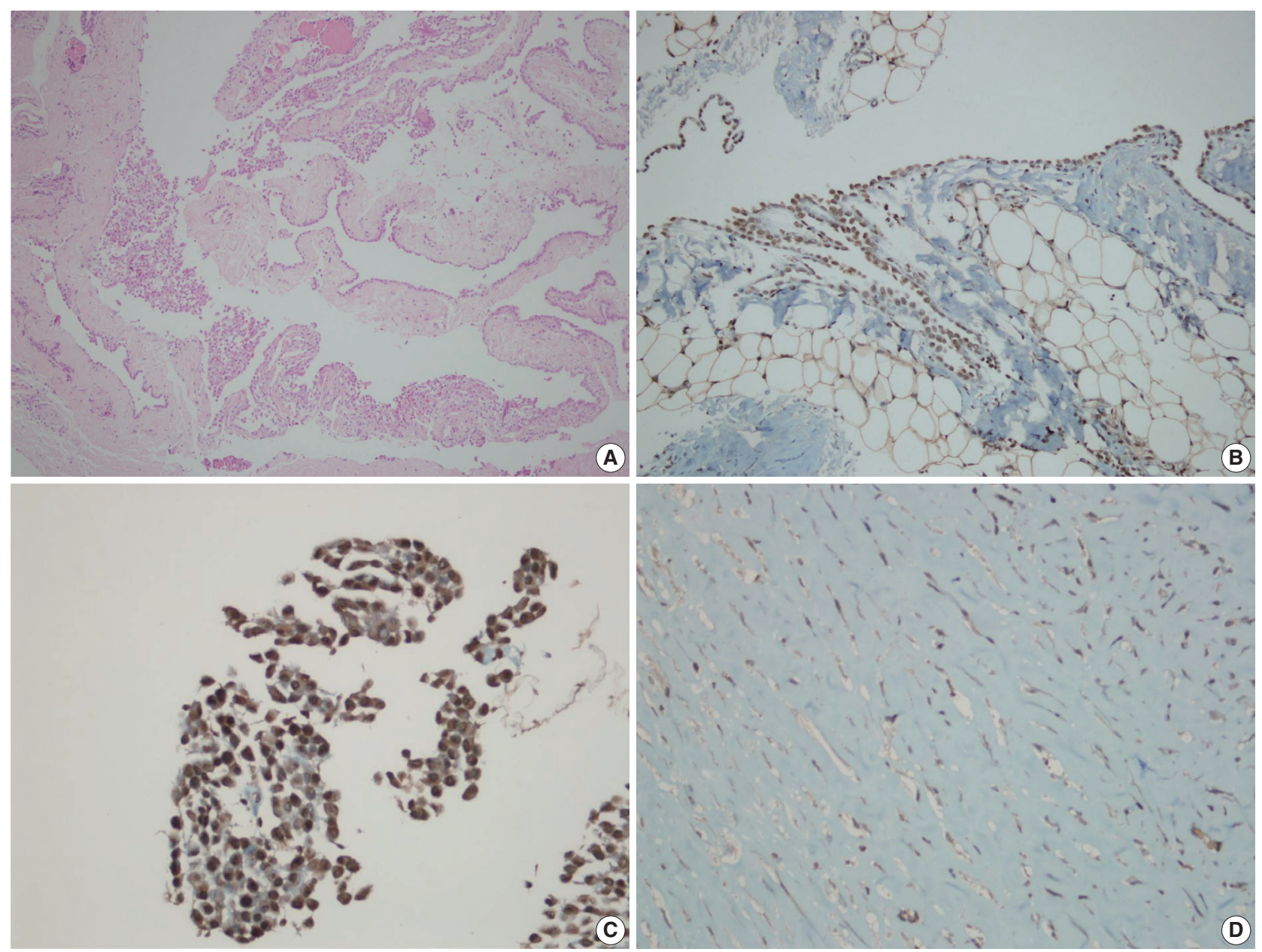

Fig. 1. BRCA1-associated protein 1 (BAP1) immunohistochemistry results in reactive mesothelial hyperplasia. Biopsy material with hematoxylin and eosin staining (A) and BAP1 immunohistochemistry (B) with strong nuclear staining. Cell block material with BAP1 immunohistochemistry staining. (C) Note the strong nuclear and weak nonspecific cytoplasmic staining. (D) An example of a fibrinous pleuritis case with BAP1 staining.

\section{(2.3\%) from the pericardium.}

According to biopsy status, the BAP1 expression loss rate (76.6\%) in patients diagnosed with MM was significantly higher than among other benign diseases $(0 \%$; $<.001)$. When MM cases were evaluated by histological subtype, the BAP1 expression loss rate was $81.5 \%$ in epithelioid-type MM cases, whereas no BAP1 expression loss was observed among sarcomatoid-type MM cases. BAP1 expression loss was observed in all five biphasic cases, but all expression loss was found in the epithelioid components, and no loss was found in the sarcomatoid components (Table 1). For MM diagnosis, the sensitivity and specificity of BAP1 expression loss in tissue biopsies were $76.56 \%$ and $100 \%$, respectively.

Only the epithelioid histological subtype of malignant mesothelioma cases had sufficient cell block material. According to cell block status, expression loss was observed in all malignant mesothelioma cases ( $\mathrm{n}=11)$, while there was no expression loss in any of the benign processes (8 RMH and $1 \mathrm{FP}$ cases). The sensitivity and specificity of the BAP1 test were both $100 \%$ for cell block (Table 2).

When the consistency between cell block and tissue biopsy results for BAP1 expression status was examined, a high consistency $(\mathrm{K}=0.90)$ and significant correlation were obtained $(\mathrm{p}<$ $.001)$. Accordingly, 10 of the 11 patients $(90.9 \%)$ who were found to have BAP1 expression loss in cell block also showed expression loss in biopsy, while all nine patients (100\%) who did not show expression loss in cell block also showed no loss in biopsy (Table 3).

MM cases were evaluated in terms of BAP1 expression loss according to age, gender, asbestos exposure, additional cancer, and localization, and no significant differences were found (all p > .05) (Table 4). 


\section{DISCUSSION}

Due to high tumor aggressiveness, distinguishing MM cases from reactive processes and diagnosing accurately as early as possible is of great importance [4-6]. However, the only reliable criterion for histopathological diagnosis is detection of fatty tissue invasion [8]. Unfortunately, these criteria cannot be evaluated in cytological materials. For this reason, numerous studies have been conducted to find an immunohistochemical marker that might aid MM diagnosis or even offer a definitive diagnosis.
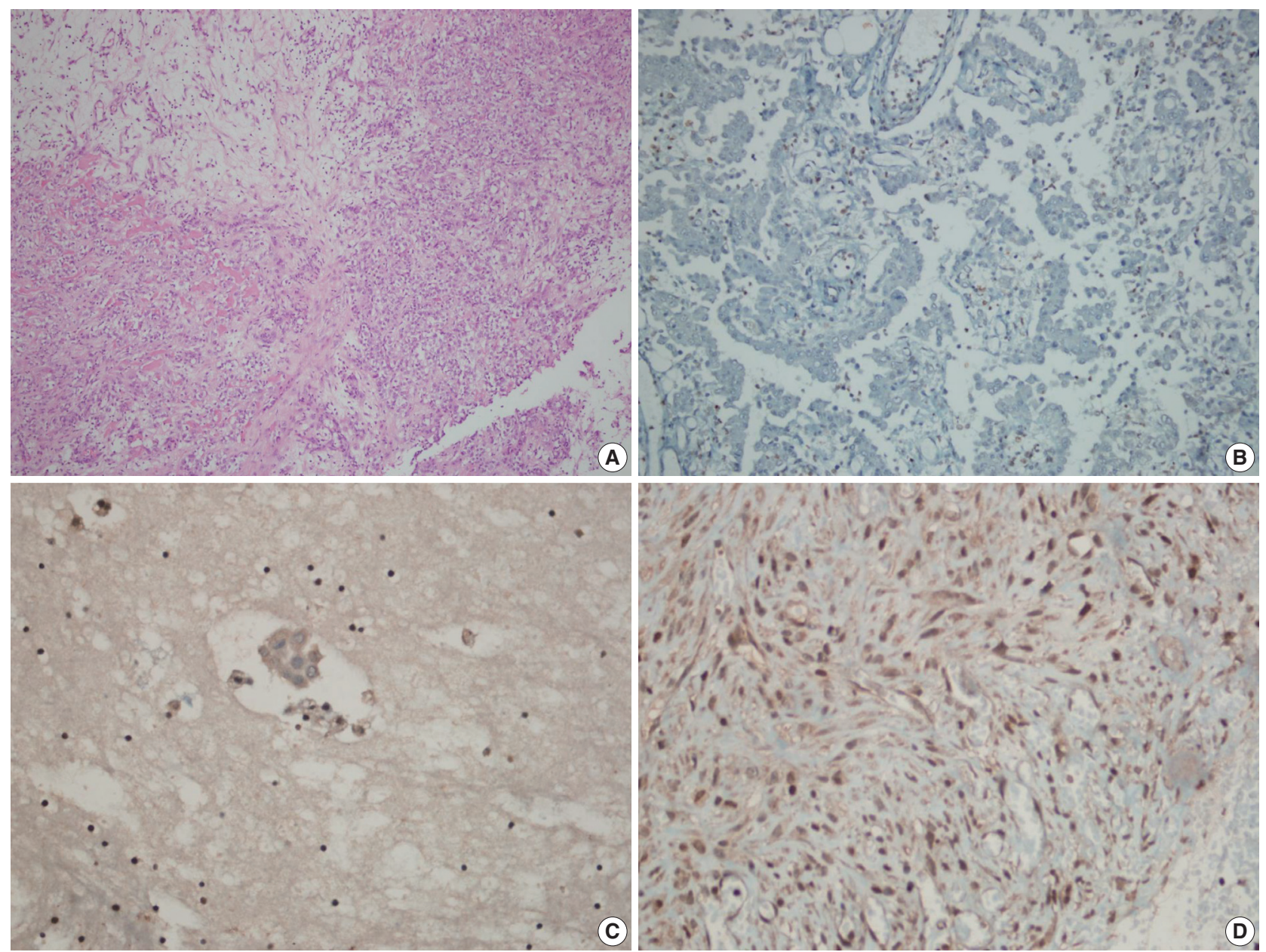

Fig. 2. BRCA1-associated protein 1 (BAP1) immunohistochemistry results in malignant mesothelioma. Biopsy material with hematoxylin and eosin staining (A) and loss of BAP1 expression (B) with inflammatory cells as a positive internal control in epithelioid mesothelioma. Cell block material with BAP1 staining (C). BAP1 normal expression in sarcomatoid mesothelioma (D).

Table 1. BAP1 expression status according to biopsy diagnosis

\begin{tabular}{lccc}
\hline & $\begin{array}{c}\text { Loss of BAP1 } \\
\text { expression } \\
(n=49)\end{array}$ & $\begin{array}{c}\text { BAP1 normal } \\
\text { expression } \\
(\mathrm{n}=152)\end{array}$ & p-value \\
\hline Histologic type $(\mathrm{n}=201)$ & $49(76.6)$ & $15(23.4)$ & $<.001$ \\
Malignant mesothelioma & 0 & $117(100)$ & \\
Reactive mesothelial hyperplasia & 0 & $20(100)$ & $<.001$ \\
Fibrinous pleuritis/Pericarditis & 0 & & \\
Histologic subtype of MM ( $\mathrm{n}=64)$ & & $10(18.5)$ & \\
Epithelioid type & $44(81.5)$ & $5(100)$ & \\
Sarcomatoid type & 0 & 0 & \\
Biphasic type & $5(100)$ & & \\
\hline
\end{tabular}

BAP1, BRCA1-associated protein 1; MM, malignant mesothelioma.
Table 2. BAP1 expression status according to cell block diagnosis

\begin{tabular}{lcccc}
\hline & $\begin{array}{c}\text { Loss of BAP1 } \\
\text { expression } \\
(n=11)\end{array}$ & $\begin{array}{c}\text { BAP1 normal } \\
\text { expression } \\
(n=9)\end{array}$ & p-value \\
\hline Histologic type $(n=20)$ & $11(100)$ & 0 & $<.001$ \\
Malignant mesothelioma & 0 & $8(100)$ & \\
Reactive mesothelial hyperplasia & 0 & $1(100)$ & \\
$\quad$ Fibrinous pleuritis/pericarditis & 0 & & $<.001$ \\
Histologic subtype of MM (n=11) & & 0 & \\
$\quad$ Epithelioid type & $11(100)$ & 0 & \\
Sarcomatoid type & 0 & 0 & \\
Biphasic type & 0 & 0 & \\
\hline
\end{tabular}

BAP1, BRCA1-associated protein 1; MM, malignant mesothelioma. 
Table 3. Consistency between BAP1 expression status in cell block and biopsy

\begin{tabular}{|c|c|c|c|}
\hline \multirow{2}{*}{$\begin{array}{l}\text { BAP1 expression status } \\
\text { in cellblock }\end{array}$} & \multicolumn{2}{|c|}{ BAP1 expression status in biopsy } & \multirow[b]{2}{*}{ Total } \\
\hline & $\begin{array}{c}\text { Loss of BAP1 } \\
\text { expression }\end{array}$ & $\begin{array}{l}\text { BAP1 normal } \\
\text { expression }\end{array}$ & \\
\hline Loss of BAP1 expression & $10(90.9)$ & $1(9.1)$ & $11(100)$ \\
\hline BAP1 normal expression & 0 & $9(100)$ & $9(100)$ \\
\hline Total & $10(50.0)$ & $10(50.0)$ & $20(100)$ \\
\hline
\end{tabular}

Values are presented as number (\%).

BAP1, BRCA1-associated protein 1. $\mathrm{p}<.001 ; \mathrm{K}=0.90$.

Table 4. BAP1 expression status according to age, sex, asbestos exposure, neoplastic transformation, and localization in MM cases

\begin{tabular}{lccc}
\hline & $\begin{array}{c}\text { Loss of BAP1 } \\
\text { expression }\end{array}$ & $\begin{array}{c}\text { BAP1 normal } \\
\text { expression }\end{array}$ & p-value \\
\hline $\begin{array}{l}\text { Age (yr) } \\
\text { Sex }\end{array}$ & $60.0(49.0-68.0)$ & $62.0(58.0-75.0)$ & .084 \\
$\quad$ Male & $29(72.5)$ & $11(27.5)$ & .322 \\
$\quad$ Female & $20(83.3)$ & $4(16.7)$ & \\
Asbestos exposure & & & \\
$\quad$ No & $32(78.0)$ & $9(22.0)$ & .708 \\
$\quad$ Yes & $17(73.9)$ & $6(26.1)$ & \\
Neoplastic transformation & & & \\
$\quad$ No & $47(75.8)$ & $15(24.2)$ & .427 \\
$\quad$ Yes & $2(100)$ & 0 & \\
Localisation & & & \\
$\quad$ Pleura & $43(75.4)$ & $14(24.6)$ & .545 \\
$\quad$ Periton and pericard & $6(85.7)$ & $1(14.3)$ & \\
\hline
\end{tabular}

Values are presented as median (percentile 25-percentile 75) or number (\%).

BAP1, BRCA1-associated protein 1; MM, malignant mesothelioma.

Various studies have demonstrated that immunohistochemical evidence of BAP1 expression loss in mesothelial cells may be a clue to malignancy. Cozzi et al. [18] reported that BAP1 expression loss in cell blocks obtained from effusions had a sensitivity of $76 \%$ for MM diagnosis, and that BAP1 expression loss in biopsy samples had $48.7 \%$ sensitivity. Meanwhile, Cigognetti et al. [19] and Walts et al. [20] estimated $100 \%$ specificity for BAP1 expression loss. In our study, we estimated $76 \%$ sensitivity and $100 \%$ specificity from biopsy tissue, but $100 \%$ sensitivity and specificity from cell blocks obtained from effusion.

Cozzi et al. [18], who investigated the role of BAP1 expression loss in effusion diagnosis for MM, found that BAP1 expression loss was $76.5 \%$ prevalent in mesothelioma cases, while it was $11.7 \%$ in mesothelial hyperplasia cases. In the same study, looking at biopsy diagnosis, BAP1 expression loss was $47.5 \%$ prevalent in patients diagnosed with mesothelioma, but no BAP1 expression loss was observed in any cases diagnosed with mesothelial hyperplasia. In our study, BAP1 expression loss was 76.6\% prevalent in biopsy materials and $100 \%$ in cell blocks. Further- more, no BAP1 expression loss was observed in either cell block or biopsy materials in our mesothelial hyperplasia cases.

There are some differences between MM subtypes in terms of nuclear BAP1 expression loss. Wu et al. [21] grouped 38 pleural MM cases as 29 biphasic and nine epithelioid types and then compared their BAP1 expression loss status and subtypes. In this study, we observed BAP1 expression loss in five of eight epithelioid-type MM cases (62.5\%), while also observing loss in both sarcomatoid and epithelioid components in five of 13 biphasic-type MM cases (38.5\%). Shinozaki-Ushiku et al. [17] studied $32 \mathrm{MM}$ cases and found BAP1 expression loss in 53\% (17/32), among which 14 were epithelioid and 3 were biphasic. Furthermore, two biphasic-type cases showed expression loss in both epithelioid and sarcomatoid components, while only 1 showed BAP1 expression persistence in the sarcomatoid component. In our study, BAP1 expression loss was observed in $81.5 \%$ of 54 epithelioid-type MM cases, whereas it was observed in all five biphasic-type MM cases (100\%), all of which were detected in the epithelioid component. Moreover, no loss of BAP1 expression was observed in any of our five sarcomatoid-type MM cases.

Massive and recurrent effusions occur in the early stages of MM. Therefore, initial diagnosis is mostly based on cytological samples, especially in the epithelioid type [22]. However, in some cases, effusion material alone is not sufficient to diagnose MM. In such cases, diagnosis requires biopsy tissue from the patients. Although the diagnostic power of biopsies have been demonstrated by numerous studies, less invasive methods are preferred by both patients and surgeons.

Pulford et al. [14] included both cytological and biopsy materials of $83 \mathrm{MM}$ cases and 18 cases with malignant pleural effusion due to metastatic adenocarcinoma. In addition to the diagnostic value and prognostic significance of BAP1 expression loss, they also measured the agreement of cytological and biopsy materials with the Kappa test. Pulford et al. [14] reported that BAP1 expression loss was observed in $59 \%$ of MM cases and the Kappa value was 0.85 . Similarly, we applied BAP1 antibodies to the biopsies of cases with cytological samples and we calculated agreement with the Kappa test. Accordingly, the agreement between cytological and histological specimens for BAP1 expression loss was excellent $(\mathrm{K}=0.90)$, and the correlation was significant $(\mathrm{p}<.001)$.

Environmental exposure to asbestos, especially for regions with abundant white soil, like some provinces of Turkey, is of great importance in MM development. Onder et al. [23] evaluated 88 MM cases from central Ankara, which is the nearest city to Cappadocia; they reported that $95 \%$ of the cases had been exposed 
to asbestos and no significant relationship was found between asbestos exposure and BAP1 expression loss. In our study, only $35.9 \%$ of $\mathrm{MM}$ cases were exposed to asbestos. We attribute this difference to the fact that the city where we work is far from Cappadocia, and we could not find as significant a relationship as Onder et al. [23] did.

There were some limitations to our work. Primarily, because of the retrospective nature of our study, it was not possible to obtain all of the cytological specimens from the biopsy materials analyzed by immunohistochemistry for BAP1 expression.

In conclusion, BAP1 expression loss was found in all the cytological materials of the cases whose diagnoses were confirmed by tissue biopsy, and the results were replicated for each case when BAP1 antibodies were applied to biopsy materials. This suggests that BAP1 expression loss is a robust criterion in achieving MM diagnosis from cytological materials. Effusion in the serosal cavity is a common early finding for MM. Furthermore, because cytologic examination is less invasive and easy to perform, it is likely to be the preferred diagnostic method, especially given the results reported here. In patients with only serous effusion and no radiological suspicion of MM but who show evidence of BAP1 expression loss by immunohistochemical analysis of cell blocks, we suggest that invasive MM may develop in the near future and, thus, these patients need strict clinical follow-up.

\section{Ethics Statement}

Approval was obtained from the Ethics Committee on May 30, 2017 (protocol number: 2017/514/108/8). Informed consent was obtained from all participants included in the study.

\section{ORCID}

Hanife Gulnihal Ozdemir Sermin Coban Kokten

https://orcid.org/0000-0003-1405-3886 Nagehan Ozdemir Barisik https://orcid.org/0000-0002-6606-1848

\section{Author Contributions}

Conceptualization: HGO, SCK. Data curation: HGO, SCK, NOB. Formal analysis: HGO. Funding acquisition: HGO, SCK, NOB. Investigation: HGO. Methodology: HGO, SCK. Supervision: HGO. Validation: HGO, NOB. Writing_original Draft: HGO, NOB. Writing-review \& editing: HGO. Approval of final manuscript: all authors.

\section{Conflicts of Interest}

The authors declare that they have no potential conflicts of interest.

\section{Funding Statement}

No funding to declare.

\section{References}

1. Cagle PT, Allen TC. Pathology of the pleura: what the pulmonologists need to know. Respirology 2011; 16: 430-8.
2. Carbone M, Bedrossian CW. The pathogenesis of mesothelioma. Semin Diagn Pathol 2006; 23: 56-60.

3. Hicks J. Biologic, cytogenetic, and molecular factors in mesothelial proliferations. Ultrastruct Pathol 2006; 30: 19-30.

4. Hida T, Hamasaki M, Matsumoto S, et al. Immunohistochemical detection of MTAP and BAP1 protein loss for mesothelioma diagnosis: comparison with 9p21 FISH and BAP1 immunohistochemistry. Lung Cancer 2017; 104: 98-105.

5. Kushitani K, Amatya VJ, Mawas AS, et al. Utility of survivin, BAP1, and Ki-67 immunohistochemistry in distinguishing epithelioid mesothelioma from reactive mesothelial hyperplasia. Oncol Lett 2018; 15: 3540-7.

6. Lee HE, Molina JR, Sukov WR, Roden AC, Yi ES. BAP1 loss is unusual in well-differentiated papillary mesothelioma and may predict development of malignant mesothelioma. Hum Pathol 2018; 79: 168-76.

7. Baris YI, Artvinli M, Sahin AA. Environmental mesothelioma in Turkey. Ann N Y Acad Sci 1979; 330: 423-32.

8. Cagle PT, Churg A. Differential diagnosis of benign and malignant mesothelial proliferations on pleural biopsies. Arch Pathol Lab Med 2005; 129: 1421-7.

9. King J, Thatcher N, Pickering C, Hasleton P. Sensitivity and specificity of immunohistochemical antibodies used to distinguish between benign and malignant pleural disease: a systematic review of published reports. Histopathology 2006; 49: 561-8.

10. Kradin RL, Mark EJ. Distinguishing benign mesothelial hyperplasia from neoplasia: a practical approach. Semin Diagn Pathol 2006; 23: 4-14.

11. Attanoos RL, Griffin A, Gibbs AR. The use of immunohistochemistry in distinguishing reactive from neoplastic mesothelium: a novel use for desmin and comparative evaluation with epithelial membrane antigen, $\mathrm{p} 53$, platelet-derived growth factor-receptor, Pglycoprotein and Bcl-2. Histopathology 2003; 43: 231-8.

12. Mayall FG, Goddard H, Gibbs AR. The frequency of p53 immunostaining in asbestos-associated mesotheliomas and non-asbestos-associated mesotheliomas. Histopathology 1993; 22: 383-6.

13. Taheri ZM, Mehrafza M, Mohammadi F, Khoddami M, Bahadori M, Masjedi MR. The diagnostic value of Ki-67 and repp86 in distinguishing between benign and malignant mesothelial proliferations. Arch Pathol Lab Med 2008; 132: 694-7.

14. Pulford E, Huilgol K, Moffat D, Henderson DW, Klebe S. Malignant mesothelioma, BAP1 immunohistochemistry, and VEGFA: does BAP1 have potential for early diagnosis and assessment of prognosis? Dis Markers 2017; 2017: 1310478.

15. McGregor SM, McElherne J, Minor A, et al. BAP1 immunohistochemistry has limited prognostic utility as a complement of CDKN2A (p16) fluorescence in situ hybridization in malignant pleural mesothelioma. Hum Pathol 2017; 60: 86-94.

16. LaFave LM, Beguelin W, Koche R, et al. Loss of BAP1 function leads to EZH2-dependent transformation. Nat Med 2015; 21: 1344-9.

17. Shinozaki-Ushiku A, Ushiku T, Morita S, Anraku M, Nakajima J, Fukayama M. Diagnostic utility of BAP1 and EZH2 expression in malignant mesothelioma. Histopathology 2017; 70: 722-33.

18. Cozzi I, Oprescu FA, Rullo E, Ascoli V. Loss of BRCA1-associated protein 1 (BAP1) expression is useful in diagnostic cytopathology of malignant mesothelioma in effusions. Diagn Cytopathol 2018; 46: 9-14.

19. Cigognetti M, Lonardi S, Fisogni S, et al. BAP1 (BRCA1-associated 
protein 1) is a highly specific marker for differentiating mesothelioma from reactive mesothelial proliferations. Mod Pathol 2015; 28: 1043-57.

20. Walts AE, Hiroshima K, McGregor SM, Wu D, Husain AN, Marchevsky AM. BAP1 Immunostain and CDKN2A (p16) FISH analysis: clinical applicability for the diagnosis of malignant mesothelioma in effusions. Diagn Cytopathol 2016; 44: 599-606.

21. Wu D, Hiroshima K, Yusa T, et al. Usefulness of p16/CDKN2A fluorescence in situ hybridization and BAP1 immunohistochemistry for the diagnosis of biphasic mesothelioma. Ann Diagn Pathol
2017; 26: 31-7.

22. Hjerpe A, Ascoli V, Bedrossian CW, et al. Guidelines for the cytopathologic diagnosis of epithelioid and mixed-type malignant mesothelioma: a secondary publication. Cytopathology 2015; 26: 14256.

23. Onder S, Ozogul E, Koksal D, Sarinc Ulasli S, Firat P, Emri S. Diagnostic value of BRCA1-associated protein-1, glucose transporter-1 and desmin expression in the discrimination between reactive mesothelial proliferation and malignant mesothelioma in tissues and effusions. Cytopathology 2019; 30: 592-600. 